\title{
Seguimiento plurianual (2013-2016) del vuelo invernal de mariposas (Lepidoptera: Papilionoidea) en el campus de la Universidad Pablo de Olavide, de Sevilla (sur de España)
}

\section{Multi-year monitoring (2013-2016) of the winter flight of butterflies (Lepidoptera: Papilionoidea) at the University Pablo de Olavide campus in Seville (southern Spain)}

\author{
DOI: $10.46932 / \mathrm{sfjdv2n5-070}$
}

Received in: Oct 1st, 2021

Accepted in: Dec 30th, 2021

\author{
Marina López-Fernández \\ Licenciada en Ciencias Ambientales. \\ Avenida de Los Pinos, 28, Mairena del Aljarafe. 41927 Sevilla (España - Spain). \\ E-mail: marina.lopez.fdez@gmail.com
}

\section{Manuel Ferreras-Romero}

Doctor en Ciencias Biológicas (PhD). Profesor titular de universidad (Professor Full), área de conocimiento Zoología.

Departamento de Sistemas Físicos, Químicos y Naturales. Universidad Pablo de Olavide, de Sevilla.

A-376 km 1, 41013 Sevilla (España - Spain)

E-mail: mferrom@upo.es

\section{RESUMEN}

Durante los inviernos 2013/2014, 2014/2015 y 2015/2016 y la primera mitad de la primavera que siguió a cada uno de ellos, fue estudiada la fenología de la fase adulta de las mariposas diurnas presentes en el campus de la Universidad Pablo de Olavide, de Sevilla (España). El número de especies observadas a lo largo de esos inviernos (12), la abundancia de cada una de ellas y los cambios en la diversidad fueron analizados. Ejemplares de Artogeia rapae, Vanessa atalanta, Vanessa cardui, Pieris brassicae, Colias crocea, Tomares ballus y Zerynthia rumina fueron observados todos los inviernos. Por último, para todas las especies halladas en este estudio ha sido realizado un estudio comparativo entre el inicio del periodo de vuelo aquí registrado y aquél que aparece recogido en la bibliografía consultada.

Palabras clave: fenología, Papilionoidea, sur de España.

\begin{abstract}
During the winters 2013/2014, 2014/2015 y 2015/2016 and early springs following each one of them, a study on phenology of diurnal butterflies existing on the campus of the University Pablo de Olavide, Seville (Spain), was carried out. Number of species recorded in those winters (12), abundance of each one of them, and changes in diversity were analyzed. Every winter specimens belonging to Artogeia rapae, Vanessa atalanta, Vanessa cardui, Pieris brassicae, Colias crocea, Tomares ballus, Zerynthia rumina were recorded. Finally, for all the species found a comparative study between the start of the flight period here registered and that appeared in bibliography was realized.
\end{abstract}

Keywords: phenology, Papilionoidea, southern Spain. 


\section{INTRODUCCIÓN}

En España están registradas 239 especies de ropalóceros y 140 de ellas se hallan en la región de Andalucía, sur de España (Fernández Haeger \& Jordano Barbudo, 2004). Los ropalóceros son usados frecuentemente como bioindicadores por su alta especificidad y sensibilidad a cambios ambientales (Geiger et al., 1987). Más importante que la abundancia de ejemplares es la diversidad de especies (Monasterio León, 2007).

En Andalucía es posible encontrar imagos volando durante todos los meses del año, incluso en pleno invierno (Fernández Haeger \& Jordano Barbudo, 2004). Las características del clima mediterráneo junto con las peculiaridades propias del valle del Guadalquivir, en el que se encuentra Sevilla, determinan importantes diferencias en la fenología de muchas especies, e incluso en el voltinismo, respecto a la desarrollada en otras zonas ibéricas y otros países europeos.

La capacidad de dispersión de las mariposas es muy variable y depende de muchas circunstancias, pero la gran mayoría sólo se desplaza decenas de metros, sin alejarse demasiado de las zonas adecuadas para depositar sus huevos y completar sus ciclos vitales (Gutiérrez et al., 2004). Las mariposas univoltinas suelen ser monófagas y las multivoltinas polífagas (Fernández Haeger et al., 2008)

\section{2 ÁREA DE ESTUDIO}

El área de estudio estuvo ubicada en parte del campus de la Universidad Pablo de Olavide, coordenadas: 37.35, -5.93. La totalidad de este campus ocupa 126 hectáreas, y está situado al sureste de la ciudad de Sevilla, a la altura del km 1 de la Ctra. Sevilla - Utrera (A-376), entre los términos municipales de Sevilla, Dos Hermanas y Alcalá de Guadaira. El diseño urbanístico original de este espacio universitario data de la década de los sesenta del pasado siglo. Presenta alternancia entre construcciones de gran volumen y extensas zonas ajardinadas, junto con zonas de aparcamiento, así como un curso de agua artificial con paredes y fondo de hormigón, de alrededor de mil metros de largo y diez de ancho. Hay diseminadas algunas pequeñas zonas con presencia de vegetación silvestre, y una gran extensión en el lado norte dedicada a uso agrícola (cultivo de trigo y girasol). La vegetación presente en el campus está recogida en Luceño et al., 2001 y Luceño Garcés et al., 2019. El arbusto más frecuente en los setos de los jardines que ocupan los espacios existentes entre los edificios es Euonymus japonicus (bonetero), y entre una amplia variedad de especies de árboles, el más común es Ligustrum lucidum (aligustrón). Es muy abundante la trepadora Aristolochia baetica (candil andaluz), y entre las herbáceas las especies más frecuentes son Piptatherum miliaceum (mijera), Thrincia hispida (diente de león), Daucus carota maximum (zanahoria silvestre) y Diplotaxis catholica (jaramago amarillo). 


\section{MÉTODOS}

El estudio de la abundancia de los distintos ropalóceros se obtuvo mediante el método de los transectos lineales, empleado con éxito desde hace muchos años por numerosos autores (e.g. Fernández Haeger \& Jordano Barbudo, 1982).

Un itinerario fue diseñado para que coincidiera con las zonas ajardinadas entre los edificios y con ambos márgenes del canal de agua; su longitud, calculada sobre una ortofoto digital, resultó ser de 3.520 m (Fig. 1). Dicho itinerario fue recorrido quincenalmente para la toma de muestras a lo largo de tres años consecutivos. Los registros de las distintas especies de mariposas fueron efectuados mayoritariamente por reconocimiento visual. El tiempo empleado en recorrer el itinerario osciló de entre 90 y 120 min.

Durante los meses de tres inviernos (entre 21 de diciembre y 21 de marzo), fueron realizados 17 transectos idénticos, en días con condiciones atmosféricas favorables, viento escaso o en calma y cielo despejado o con poca nubosidad, y en las horas diurnas en las que las mariposas se muestran más activas, entre las 13 y las 16, hora oficial española. La totalidad de las visitas fueron efectuadas coincidiendo con días sin actividad académica, para minimizar la presencia de personas y vehículos en la zona estudiada. El intervalo entre dos muestreos consecutivos fue en torno a 15 días, siempre que las condiciones climatológicas lo permitieron; cuando las condiciones atmosféricas fueron desfavorables fue aplazada la realización del transecto hasta el siguiente fin de semana favorable.

El objetivo principal del estudio fue conocer la composición de la comunidad de mariposas durante el periodo invernal. Sin embargo, los tres años la toma de registros continuó durante la primera mitad de la primavera, con el fin de saber la respuesta temprana de las especies a la llegada de esta otra estación más templada.

Durante los primeros recorridos efectuados y siempre que fue observada una especie nueva, los individuos fueron capturados con una manga entomológica aérea para su identificación, utilizando las guías de Higgins \& Riley (1973) y Tolman \& Lewington (2011), siendo liberados pocos minutos después, siempre en el lugar de la captura. En las sucesivas tomas de datos posteriores fueron identificados visualmente sin necesidad de captura. Las familias consideradas en este estudio son las aceptadas en la obra de Tolman \& Lewington (2011): Papilionidae, Pieridae, Lycaenidae, Nymphalidae, Satiridae y Hesperiidae.

\section{RESULTADOS Y DISCUSIÓN}

\subsection{INVIERNOS}

Los registros de mariposas efectuados durante los meses de invierno fueron más de 50 todos los años, osciló entre 52 y 62 (Tabla 1). La media de observaciones de mariposas por transecto realizado fue 10,0; osciló entre 8,6 (invierno 2014/2015) y 11,2 (invierno 2015/2016). Siete especies fueron observadas 
todos los inviernos, y acumulan más del $90 \%$ de los 170 registros invernales obtenidos (91,2\%), y el conjunto de tres de ellas (Artogeia rapae, Vanessa cardui y Pieris brassicae) suponen más del 50\% de los registros invernales (Tabla 2). La especie más constante fue A. rapae, observada en 14 de los 17 transectos realizados. Menos constantes fueron Zerynthia rumina y Tomares ballus, ya que todos sus registros fueron obtenidos a partir de febrero.

A estas siete especies se añaden algunos inviernos Papilio machaon (observaciones siempre a partir de mitad de febrero, y ningún registro en 2015/2016), Lycaena phlaeas y Euchloe belemia (ningún registro en 2014/2015). Otras dos especies, Gonepteryx cleopatra y Euchloe tagis, solamente fueron observadas uno de los tres inviernos estudiados.

En la campaña 2013/14 el número de especies registrado en los seis muestreos de invierno osciló entre tres, a finales de diciembre, y ocho, a primeros de marzo; el número acumulado de especies fue once al finalizar el invierno (Fig. 2). Este primer invierno el número acumulado de especies observadas mostró una clara tendencia ascendente sostenida a lo largo del periodo invernal, siendo frecuente la observación de seis o siete especies distintas en cada día de toma de datos.

Las campañas 2014/15 y 2015/16 mostraron tendencias muy diferentes en cuanto al número de especies que iban siendo observadas a lo largo del periodo invernal. En el conjunto de los tres primeros muestreos de la campaña 2014/15 sólo fueron registradas dos especies, A. rapae y V. cadui. Esa escasa riqueza observada en la primera mitad de este invierno parece estar relacionada con temperaturas mínimas bajas (Fig.3), una media de mínimas cuatro grados inferior a la del año anterior que pudieron retrasar la aparición de las mariposas. Estas bajas temperaturas nocturnas deben estar relacionadas con las escasas precipitaciones registradas, especialmente en febrero de 2015 (Fig.4), y por consiguiente con un elevado número de noches con el cielo despejado de nubosidad. Los inviernos con escasas precipitaciones, o concentradas en pocos días, y numerosas noches despejadas y frías retrasan la aparición de las especies con vuelo invernal.

Durante los tres inviernos que duró este estudio, el valor del índice de diversidad de ShannonWeaver de la mayoría de las muestras osciló entre 1,5 y 2,5 (Tabla 1, Fig. 5). En este estudio, los valores más bajos de diversidad (cercanos a 1,0) fueron obtenidos en la primera mitad de invierno, aumentando a medida que avanzaba la estación, hasta alcanzar valores próximos o superiores a 2,0 a final de invierno. La diversidad ecológica a finales del invierno de 2014 fue excepcionalmente alta, con un valor del índice cercano a 3,0. Estos valores de diversidad de ropalóceros obtenidos a final de invierno, en contra de lo que se podría esperar, no fueron superados por los obtenidos en las muestras de primavera temprana. 


\subsection{PRIMAVERAS TEMPRANAS}

Los registros efectuados cada año en la primera mitad de la primavera incrementaron el inventario en solamente cuatro especies, cada una de ellas observadas en una única ocasión: Polyommatus icarus (8.IV.2014), Carcharodus boeticus (27.IV.2014), Pararge aegeria (23.III.2014) y Leptotes pirithous (24.IV.2016).

En dos de los años en que se llevó a cabo este estudio, 2015 y 2016, el incremento esperable de la diversidad durante la primavera temprana fue muy escaso. Por el contrario, en el año 2014 el aumento en la riqueza respecto al invierno precedente fue notable, hubo un incremento de cuatro especies respecto al invierno previo, pasando de once a quince especies $(\Delta=36,4 \%)$. Las dos primaveras siguientes el incremento fue de sólo una especie $(\Delta=11,1 \%)$. Esta diferencia en el incremento de la riqueza no resulta fácil de explicar a partir de los datos climáticos de esos años. Mientras que la evolución de la media mensual de temperaturas mínimas primaverales fue muy similar los tres años (Fig. 5), las precipitaciones fueron muy similares en las primaveras de 2014 y 2015, y más abundantes en abril y mayo de 2016 (Fig. 4).

En seis especies fueron constatadas notables diferencias fenológicas (Tabla 3) entre los periodos recogidos en Tolman \& Lewington (2011) y las observaciones realizadas en este estudio (ver Anexo).

Asumiendo la innegable variabilidad interanual intrínseca al clima mediterráneo, como consecuencia del actual proceso de calentamiento global podría ser esperable un incremento progresivo del número de especies cuyo periodo de vuelo comenzase todos los años durante el inverno; en este estudio sólo siete especies. También parece esperable el adelanto del periodo de vuelo de algunas de las especies que en los años aquí considerados no fueron observadas hasta la primavera temprana. Sin embargo, el régimen de temperaturas mínimas invernales está muy condicionado por la nubosidad y las correspondientes precipitaciones de lluvia. Si se produce un incremento en las temperaturas máximas registradas en los días del invierno, pero a la vez disminuye la llegada de frentes de lluvia desde el Atlántico y las mínimas nocturnas resultan ser incluso inferiores a las actuales, número de especies con vuelo invernal en los años futuros resulta imprevisible. 


\section{REFERENCIAS}

Fernández Haeger, J. \& D. Jordano Barbudo (1982). Fenología de una comunidad mediterránea de mariposas diurnas. Biologie-Ecologie Méditerranéenne, 9 (1): 19-28.

Fernández Haeger, J. \& D. Jordano Barbudo (2004). Las mariposas del monte mediterráneo andaluz. En: Herrera, C. M. (coordinador). El monte mediterráneo en Andalucía. Consejería de Medio Ambiente, Junta de Andalucía, Sevilla: 61-69.

Fernández Haeger, J., D. Jordano, J. L. Reyes \& G. Luque (2008). Restauración y seguimiento de las comunidades de mariposas del Corredor Verde del Guadiamar. En: Montes del Olmo, C. \& F. Carrascal Moreno (coordinadores). La restauración ecológica del Guadiamar y el proyecto del Corredor Verde. Consejería de Medio Ambiente, Junta de Andalucía, Sevilla: 343-356.

Geiger, W., C. Dufour \& Y. Gonseth (1987). Le Centre Suisse de cartographie de la faune (CSCFNeuchâtel) et la protection des espèces en Suisse. Revue suisse de Zoologie, 94 (3): 511-514.

Gutiérrez, D., A. Seymour, P. Fernández, J. Fernández Haeger \& D. Jordano (2004). Estructura espacial y dispersión en poblaciones de mariposas: modelos y experimentos con Plebejus argus en Doñana. En: Fernández - Palacios, J.M. \& C. Morici (Eds). Ecología Insular. Asociación Española de Ecología Terrestre: 147-179.

Higgins, L.G. \& N.D. Riley (1973) Guía de campo de las mariposas de España y de Europa. Omega, Barcelona: $392 \mathrm{pp}$.

Luceño, M., S. Martín \& J. Solís (2001). Plantas silvestres de Campus de la Universidad Pablo de Olavide. Consejería de Medio Ambiente de la Junta de Andalucía y Universidad Pablo de Olavide, Sevilla. 192 pp.

Luceño Garcés, M., S. Martín Bravo, R. Sánchez Villegas, M. Escudero Lirio, M. Sánchez Villegas, J.I. Márquez Corro \& I. Pulgar Sañudo (2019). Plantas Silvestres y Ornamentales de Campus de la Universidad Pablo de Olavide. Fundación Universidad Pablo de Olavide, Sevilla. 446 pp.

Monasterio León, Y. (2007). Los lepidópteros de La Rioja. Páginas de Información Ambiental, 25: 2428.

Tolman, T. \& R. Lewington (2011). Guía de mariposas de España y Europa. Lynx Edicions, Barcelona: $384 \mathrm{pp}$. 


\section{TABLAS}

Tabla 1.- Número de registros invernales, media de observación / transecto, riqueza de especies y valores de diversidad por campaña y media de la diversidad de las muestras y rango (entre paréntesis).

\begin{tabular}{|c|c|c|c|c|}
\hline Campañas & $2013-2014$ & $2014-2015$ & $2015-2016$ & $\begin{array}{c}\text { Total / } \\
\text { Media } \\
\text { interanual }\end{array}$ \\
\hline $\mathrm{N}^{\mathbf{o}}$ de registros en invierno & 62 & 52 & 56 & 170 \\
\hline $\begin{array}{c}\text { Media de observaciones / } \\
\text { transecto de invierno }\end{array}$ & 10,3 & 8,6 & 11,2 & 10,0 \\
\hline $\begin{array}{c}\mathrm{N}^{\mathbf{o}} \text { de especies observadas } \\
\text { en invierno }\end{array}$ & 11 & 9 & 9 & 12 \\
\hline $\begin{array}{c}\text { Diversidad anual invernal } \\
\text { (índice de Shannon- } \\
\text { Weaver) }\end{array}$ & 3,11 & 2,77 & 2,87 & 1,70 \\
\hline $\begin{array}{c}\text { Media de diversidad en las } \\
\text { muestras de invierno } \\
\text { (rango) }\end{array}$ & $\begin{array}{c}2,22 \\
(1,5-2,85)\end{array}$ & $\begin{array}{c}1,25 \\
(0-2,36)\end{array}$ & $\begin{array}{c}1,64 \\
(0,81-2,53)\end{array}$ & 1,81 \\
\hline $\begin{array}{c}\text { Media de diversidad en las } \\
\text { muestras de invierno y } \\
\text { primavera temprana } \\
\text { (rango) }\end{array}$ & $\begin{array}{c}2,31 \\
(1,5-2,98)\end{array}$ & $(0-2,36)$ & $(0,81-2,53)$ & 1,71 \\
\hline
\end{tabular}

Tabla 2.- Especies observadas todos los inviernos. Se muestran constancia en la observación de la especie (\% de los 17 muestreos invernales en los que aparece) y frecuencia de observaciones invernales (\% que aporta la especie en los 170 registros obtenidos).

\begin{tabular}{|l|c|c|}
\hline Especies & Constancia (17 transectos) & Frecuencia (170 registros) \\
\hline Artogeia rapae & 82,4 & 17,65 \\
\hline Vanessa atalanta & 58.8 & 11.76 \\
\hline Vanessa cardui & 52.9 & 15.29 \\
\hline Pieris brassicae & 52.9 & 18.24 \\
\hline Colias crocea & 52.9 & 8.24 \\
\hline Tomares ballus & 41.2 & 10.59 \\
\hline Zerynthia rumina & 29.4 & 9.41 \\
\hline & & $\Sigma=91,18$ \\
\hline
\end{tabular}

Tabla 3.- Diferencias fenológicas entre los periodos recogidos en Tolman \& Lewington (2011) y las observaciones realizadas en este estudio (ver Anexo).

\begin{tabular}{|l|c|l|}
\hline Especies & $\begin{array}{l}\text { Periodo de vuelo según Tolman \& } \\
\text { Lewington (2011) }\end{array}$ & Observaciones propias \\
\hline Artogeia rapae & marzo - noviembre & Presente durante todo el invierno \\
\hline Colias crocea & marzo - noviembre & $\begin{array}{l}\text { Constante todos los años desde la } \\
\text { segunda quincena de febrero }\end{array}$ \\
\hline Vanessa atalanta & junio - octubre & $\begin{array}{l}\text { Presente durante todo el invierno, } \\
\text { excepto los más fríos }\end{array}$ \\
\hline Zerynthia rumina & final de marzo - mayo & $\begin{array}{l}\text { Suele aparecer a mitad de febrero, } \\
\text { adelantándose casi mes y medio }\end{array}$ \\
\hline Pieris brassicae & marzo - octubre & $\begin{array}{l}\text { Suele estar presente desde mitad de } \\
\text { febrero, aunque pueden comenzar las } \\
\text { observaciones a finales de enero }\end{array}$ \\
\hline Euchloe tagis & $\begin{array}{l}\text { Un año fue observada a principios de } \\
\text { marzo }\end{array}$ \\
\hline
\end{tabular}




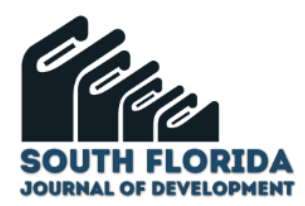

\section{FIGURAS}

Fig.1.- Itinerario diseñado en el campus de la Universidad pablo de Olavide, de Sevilla (España), para llevar a cabo los transectos efectuados en este estudio: I, inicio; F, final, está indicado con flechas el sentido de marcha.

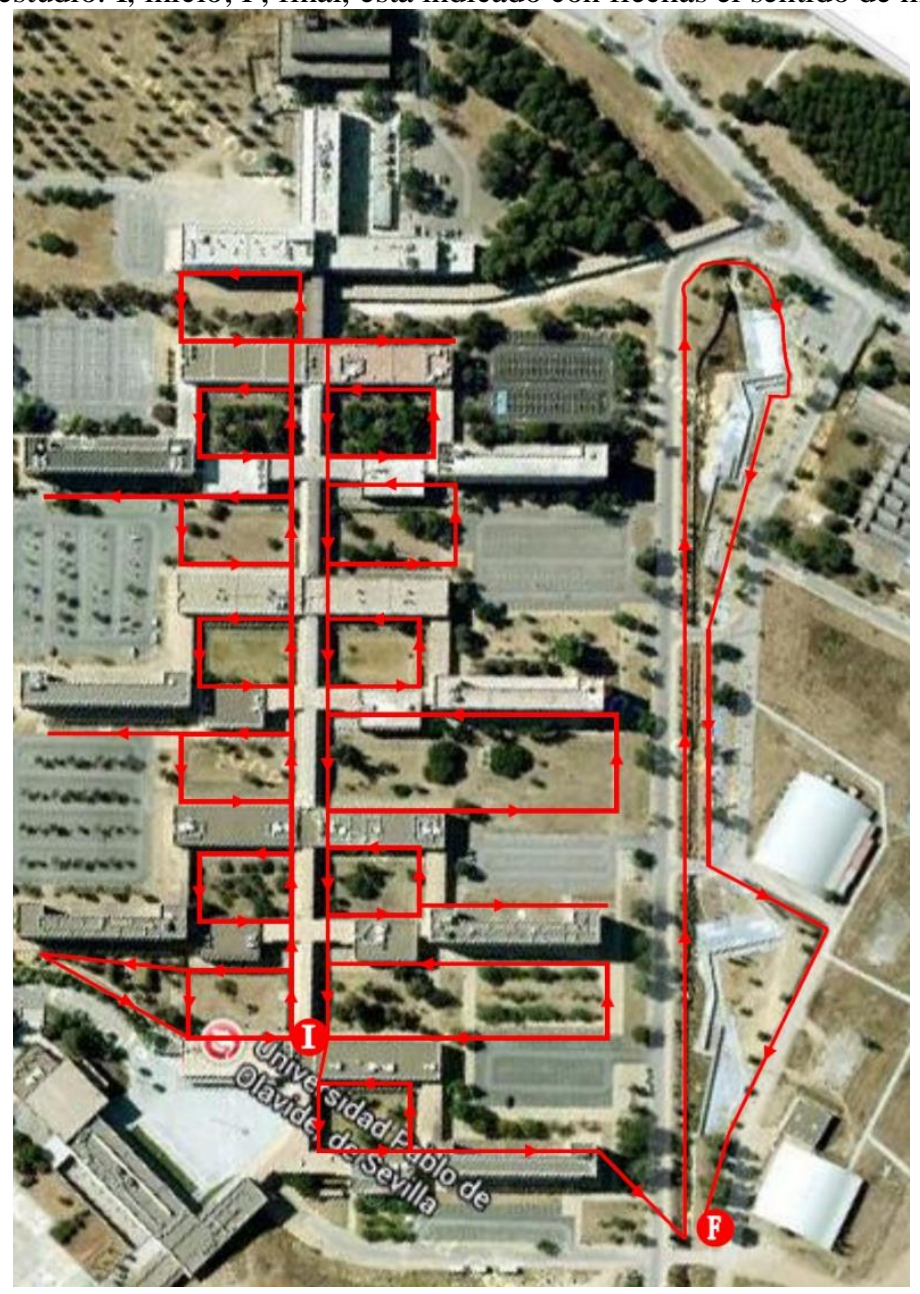

Fig. 2.- Número acumulado de especies registradas en los tres inviernos (muestreos 1 a 6 ) y primaveras tempranas (muestreos 7 a 9) en que fue realizado este estudio.

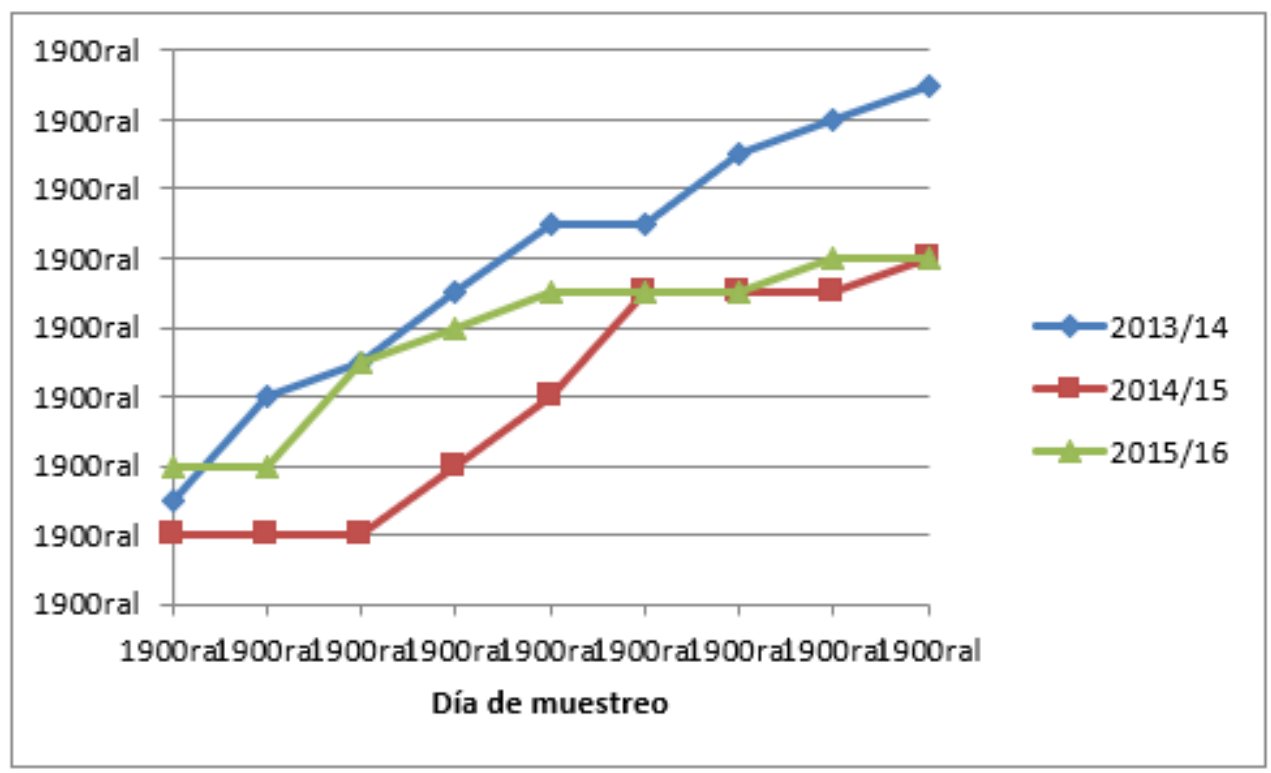


Fig. 3.- Registro de temperaturas mínimas mensuales en el aeropuerto de Sevilla (San Pablo) en los tres años en que fue realizado este estudio.

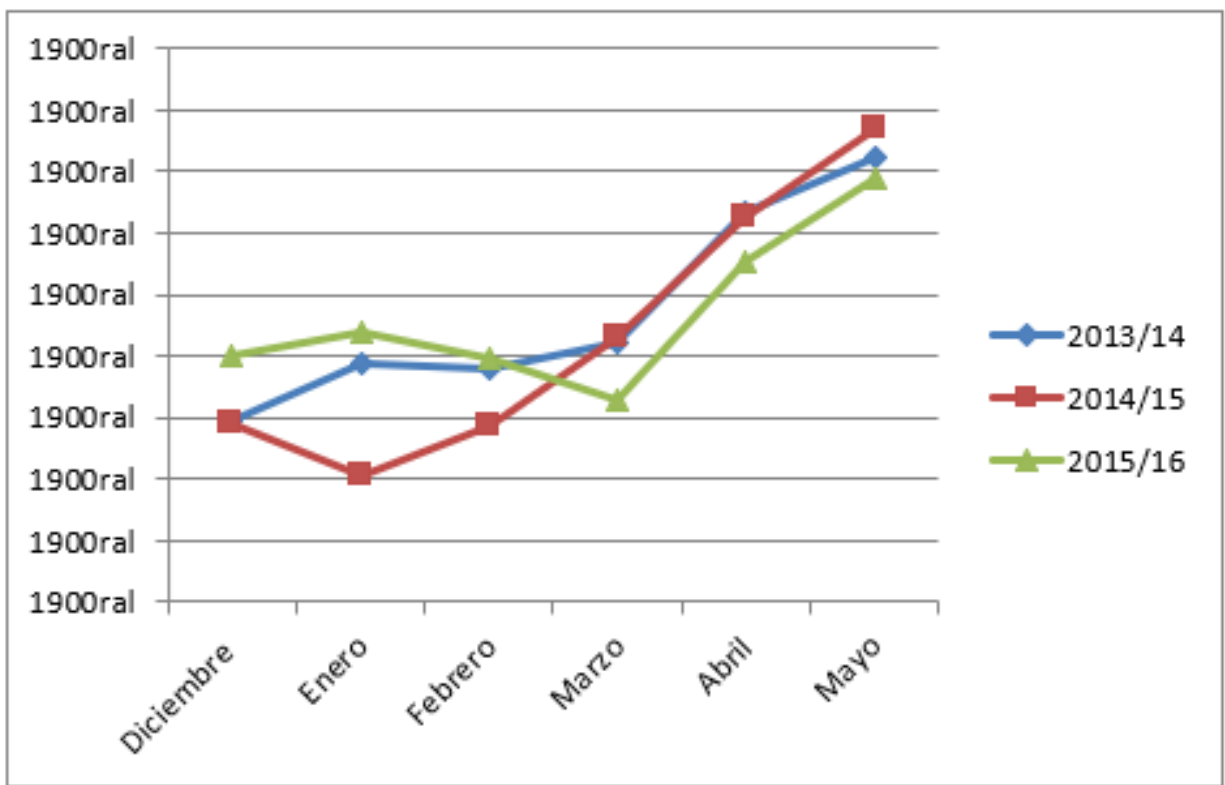

Fig. 4.- Registro de precipitaciones mensuales en el aeropuerto de Sevilla (San Pablo) en los tres años en que fue realizado este estudio.

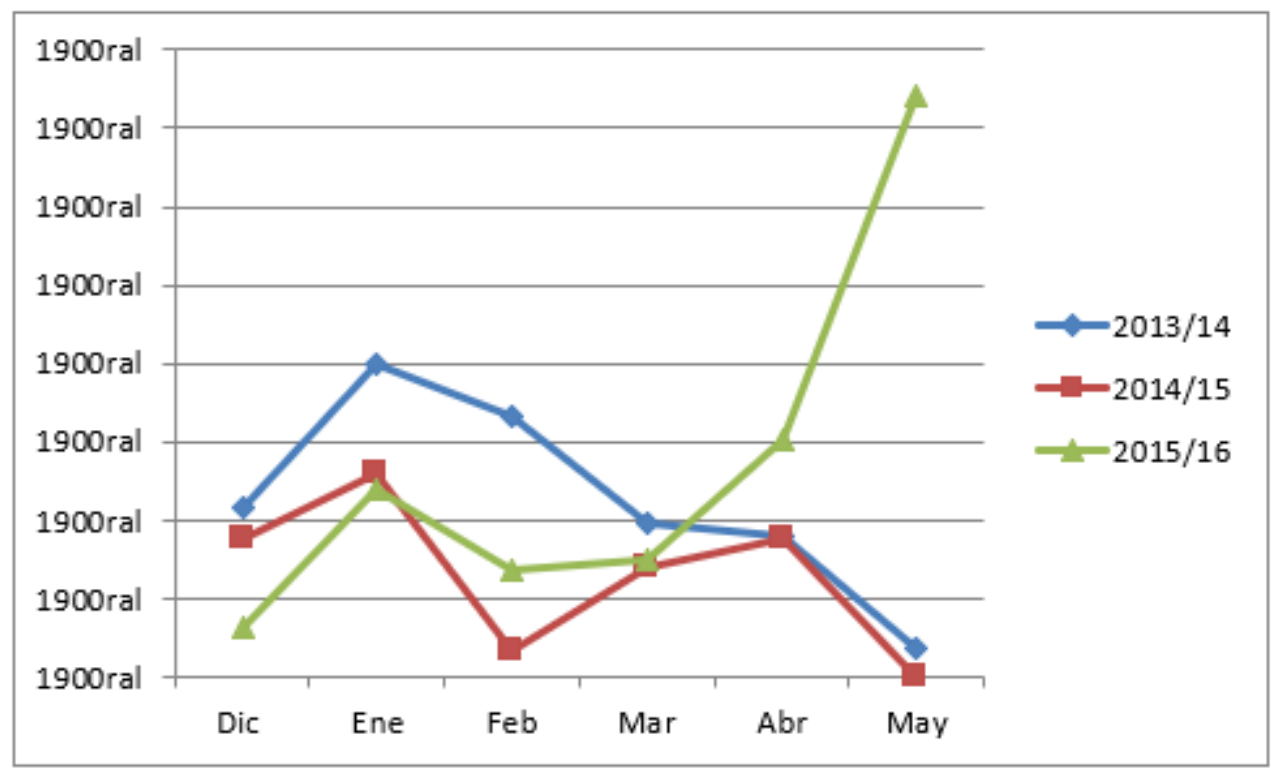


Fig. 5. Valores mensuales de diversidad según el índice de Shannon-Weaver

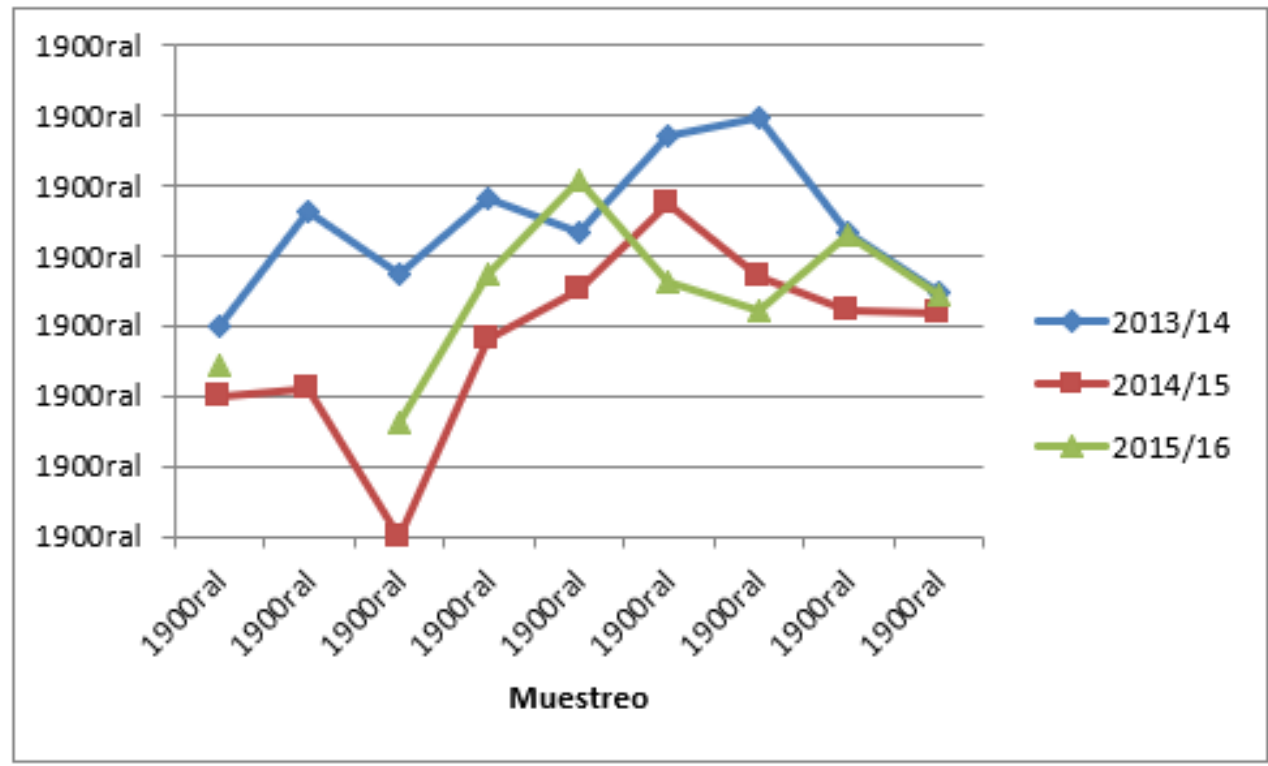

Anexo. Fechas de las observaciones de las seis especies que muestran diferencias fenológicas evidentes respecto a los periodos de vuelo recogidos en Tolman \& Lewington (2011).

\begin{tabular}{|c|c|c|c|c|c|c|c|c|c|}
\hline A.rapae & & & & & & & & & \\
\hline $13 / 14$ & $28 . \mathrm{XII}$ & $11 . \mathrm{I}$ & $26 . \mathrm{I}$ & $16 . \mathrm{II}$ & $22 . \mathrm{II}$ & $8 . \mathrm{III}$ & $23 . \mathrm{III}$ & $8 . \mathrm{IV}$ & $27 . \mathrm{IV}$ \\
\hline $14 / 15$ & $3 . \mathrm{I}$ & $10 . \mathrm{I}$ & $25 . \mathrm{I}$ & $22 . \mathrm{II}$ & & $14 . \mathrm{III}$ & $23 . \mathrm{III}$ & $12 . \mathrm{IV}$ & $3 . \mathrm{V}$ \\
\hline $15 / 16$ & $27 . \mathrm{XII}$ & - & $24 . \mathrm{I}$ & & & $13: \mathrm{III}$ & $14 . \mathrm{IV}$ & $24 . \mathrm{IV}$ & $2 . \mathrm{V}$ \\
\hline
\end{tabular}

\begin{tabular}{|c|c|c|l|l|l|l|l|l|l|}
\hline C.crocea & & & & & & & & & \\
\hline $13 / 14$ & & $11 . \mathrm{I}$ & & & $22 . \mathrm{II}$ & 8.III & $23 . \mathrm{III}$ & $8 . \mathrm{IV}$ & \\
\hline $14 / 15$ & & & & $22 . \mathrm{II}$ & $1 . \mathrm{III}$ & $14 . \mathrm{III}$ & $23 . \mathrm{III}$ & $12 . \mathrm{IV}$ & \\
\hline $15 / 16$ & $27 . \mathrm{XII}$ & - & & & $28 . \mathrm{II}$ & $13 . \mathrm{III}$ & $14 . \mathrm{IV}$ & $24 . \mathrm{IV}$ & $2 . \mathrm{V}$ \\
\hline
\end{tabular}

\begin{tabular}{|c|c|c|c|c|c|c|c|c|c|}
\hline V.atalanta & & & & & & & & & \\
\hline $13 / 14$ & $28 . X I I$ & $11 . I$ & $26 . I$ & $16 . I I$ & $22 . I I$ & & $23 . I I I$ & $8 . I V$ & \\
\hline $14 / 15$ & & & & & & 14. III & $23 . I I I$ & $12 . I V$ & \\
\hline $15 / 16$ & $27 . X I I$ & - & 24.I & 20.II & 28.II & 13:III & & & \\
\hline
\end{tabular}

\begin{tabular}{|c|l|l|l|l|l|l|l|l|l|}
\hline Z.rumina & & & & & & & & & \\
\hline $13 / 14$ & & & & 16. II & & 8. III & 23. III & 8. IV & \\
\hline $14 / 15$ & & & & & & 14. III & & & \\
\hline $15 / 16$ & & & & $20 . I I$ & $28 . I I$ & & $14 . I V$ & $24 . I V$ & \\
\hline
\end{tabular}

\begin{tabular}{|c|l|l|l|l|l|l|l|l|l|}
\hline P.brassicae & & & & & & & & & \\
\hline $13 / 14$ & & & $26 . I$ & $16 . \mathrm{II}$ & $22 . \mathrm{II}$ & $8 . \mathrm{III}$ & $23 . \mathrm{III}$ & $8 . \mathrm{IV}$ & $27 . \mathrm{IV}$ \\
\hline $14 / 15$ & & & & & $1 . \mathrm{III}$ & $14 . \mathrm{III}$ & $23 . \mathrm{III}$ & $12 . \mathrm{IV}$ & $3 . \mathrm{V}$ \\
\hline $15 / 16$ & & & & $20 . \mathrm{II}$ & $28 . \mathrm{II}$ & 13 III & $14 . \mathrm{IV}$ & $24 . \mathrm{IV}$ & $2 . \mathrm{V}$ \\
\hline
\end{tabular}

\begin{tabular}{|c|l|l|l|l|l|l|l|l|l|}
\hline E.tagis & & & & & & & & & \\
\hline $13 / 14$ & & & & & & & 23. III & & \\
\hline $14 / 15$ & & & & & 1. III & & & & \\
\hline $15 / 16$ & & & & & & & & & \\
\hline
\end{tabular}

